\title{
Real-Time Light-Guided Vocal Fold Injection: An In Vivo Feasibility Study in a Canine Model
}

\author{
Hee Young Son ${ }^{1}$ (i) $\cdot$ Sanghoon Kim² (i) $\cdot$ Ramla Talib Mohammad ${ }^{3}$ (i) $\cdot$ Gene $\mathrm{Huh}^{3}$ (i) \\ Hyojin Kim ${ }^{4}$ (i) $\cdot$ Woo-Jin Jeong ${ }^{3}$ (i) $\cdot$ Wonjae Cha $^{3}$ (i) \\ 'Department of Otorhinolaryngology-Head and Neck Surgery, The Dongnam Institute of Radiological and Medical Sciences (DIRAMS), Busan; \\ ${ }^{2}$ Department of Otorhinolaryngology-Head and Neck Surgery and Biomedical Research Institute, Pusan National University Hospital, Busan; \\ Departments of ${ }^{3}$ Otorhinolaryngology-Head and Neck Surgery and ${ }^{4}$ Pathology, Seoul National University Bundang Hospital, Seoul National \\ University College of Medicine, Seongnam, Korea
}

Objectives. The transcutaneous approach is a good option for office-based vocal fold injection (VFI). However, precise localization requires extensive experience because the needle tip is invisible in small and complex laryngeal spaces. Recently, real-time light-guided VFI (RL-VFI) was proposed as a new technique that allows simultaneous injection under precise needle localization by light guidance. Herein, we aimed to verify the feasibility of RL-VFI in an in vivo canine model and explored its clinical usefulness.

Methods. The device for RL-VFI comprised a light source (light-emitting diode modules [10 W] of red color [650 nm]) and injectors (1.5 inches, 23 gauge). An adult male beagle was used for the experiment. After tracheostomy, a rigid laryngoscope was inserted and suspended to expose the larynx. A flexible naso-laryngoscopy system was used to visualize the vocal folds.

Results. RL-VFI was performed using various transcutaneous approaches, including the cricothyroid, transthyroid, and transhyoid approaches. Light guidance helped identify the path of the needle and prevent inadvertent penetration. The location of the needle tip was accurately indicated by the light. The illuminated needle could be easily placed at the intended points in the vocal fold with real-time visual-motor feedback. Hyaluronic acid could be simultaneously injected lateral to the vocal process under light guidance without manipulation of the device.

Conclusion. RL-VFI was found to be safe and feasible in an in vivo canine model, providing precise localization and visualmotor feedback. The clinical application of RL-VFI is expected to improve the safety and precision of VFI.

Keywords. Vocal Fold Injection; Real-Time Light-GuidedVocal Fold Injection; InVivo Animal Study; Transcutaneous Approach; Vocal Fold Palsy

\section{INTRODUCTION}

Vocal fold injection (VFI) is a laryngological procedure that delivers drugs or materials to the vocal folds through a needle and has a history of over 100 years [1,2]. It can be used to treat vari-

\footnotetext{
- Received October 25, 2020

Accepted December 1, 2020

- Corresponding author: Wonjae Cha

Departments of Otorhinolaryngology-Head and Neck Surgery, Seoul

National University Bundang Hospital, Seoul National University College

of Medicine, 82 Gumi-ro 173beon-gil, Bundang-gu, Seongnam 13620,

Korea

Tel: +82-31-787-7413, Fax: +82-31-787-4057

E-mail: chawonjae@gmail.com
}

ous vocal fold pathologies, including vocal fold atrophy, vocal fold scarring, vocal fold papillomatosis, vocal fold nodules, vocal fold polyps, vocal fold granuloma, Reinke edema, and vocal fold paralysis (VFP) [1,2]. Recently, this minimally invasive technique has been re-highlighted in laryngology with advances in materials science and endoscopic technology $[2,3]$.

Currently, the approaches of VFI are classified according to the route of injection as transcutaneous (transcricothyroid [CT] membrane, transthyroid [TT] cartilage and transhyoid [TH] membrane approaches), transoral, and transnasal approaches [2]. The choice of the approach usually depends on the anatomical characteristics of patients, the injection material, and the surgeon's preference [4-6]. The transoral and CT approaches are

Copyright @ 2021 by Korean Society of Otorhinolaryngology-Head and Neck Surgery.

This is an open-access article distributed under the terms of the Creative Commons Attribution Non-Commercial License (https://creativecommons.org/licenses/by-nc/4.0)

which permits unrestricted non-commercial use, distribution, and reproduction in any medium, provided the original work is properly cited. 
the most commonly used approaches in current practice. The transoral approach is very intuitive and easy to understand because of the good localization of the needle tip [2,4]. However, it is difficult to handle the long-curved needle delicately during the transoral approach, and mucosal penetration may lead to spillage of the injectate, bleeding, and laryngeal spasm [2,7]. In comparison with the transoral approach, the $\mathrm{CT}$ approach is advantageous due to good patient compliance, suitability as an office-based procedure, and a low complication rate [6-12].

However, despite its advantages, the CT approach has the fundamental limitation that precise localization of the needle tip is very difficult because it moves inside the vocal fold $[4,5]$. Most laryngologists usually estimate the location of the needle tip indirectly based on the distortion of the vocal fold configuration. Therefore, a high level of experience is necessary to perform VFI with the CT approach proficiently, and mastery of this technique involves a steep learning curve $[13,14]$. In South Korea, only a few laryngologists at referral hospitals perform the majority $(90 \%)$ of VFI cases [15].

Recently, Cha et al. $[4,5]$ suggested that the invisibility of the needle tip is the primary reason for the technical limitations of the CT approach, and proposed a new technique to overcome the invisibility of the needle, which they termed real-time lightguided VFI (RL-VFI); it allows simultaneous injection under precise needle localization by light guidance. In previous studies, conceptual models for RL-VFI were developed, and its technical feasibility was validated in an ex vivo canine larynx model $[4,5]$. The RL-VFI device could intuitively provide visual information on the precise location of the needle tip by light guidance during VFI.

However, in real office-based VFI, the vocal folds should be visualized by a flexible laryngoscope through the dark nasooropharyngeal tract, and the needle passes through more complex anatomical layers from the skin to the vocal fold. The clinical setting is therefore somewhat different from that of previous studies, which used extracted larynges. Notwithstanding the promising previous results, experiences of RL-VFI in ex vivo models do not provide a sufficient basis for conducting a clinical

\section{H I G G H L I G G H T S}

- Real-time light-guided vocal fold injection (RL-VFI) provided precise localization and visual-motor feedback in an in vivo animal model.

- RL-VFI would be helpful in various transcutaneous approaches, including the cricothyroid approach, the transthyroid approach, and the thyrohyoid approach.

- The clinical application of RL-VFI is expected to improve safety and precision in VFI.

- This study establishes the rationale for an upcoming clinical trial. trial. In this study, we aimed to verify the feasibility of RL-VFI in an in vivo canine model with various transcutaneous approaches and to establish a rationale for conducting a clinical trial in humans.

\section{MATERIALS AND METHODS}

\section{Ethical consideration}

The animal care and use protocol of this study was reviewed and approved by the Institutional Animal Care and Use Committee at Pusan National University Yangsan Hospital (No. PNUYH2018-126) and Seoul National University Bundang Hospital (No. BA-2101-312-004-01), and all methods were carried out in accordance with approved guidelines.

\section{The device for RL-VFI}

The device for RL-VFI was developed for this experiment. The device comprised two components of the light source and the injectors. The light source had light-emitting diode modules (10 W) of red color $(650 \mathrm{~nm})$ and emitted light via a single optic fiber. The injectors consisted of a needle (1.5 inch, 23 gauge) and a connector with an optic fiber cable (Fig. 1A and B).

\section{Animal model and experimental setting}

A 10-month-old adult male beagle (Hana Bio Technology, Pyeongtaek, Korea) weighing $13 \mathrm{~kg}$ was used for the experiment. The dog was humanely anesthetized by the veterinarian. After tracheostomy, a rigid laryngoscope was inserted and suspended to expose the larynx.

A full high-definition video-laryngoscopy system consisted of a video processor (DEFINA, EPK-3000; PENTAX Medical, Tokyo, Japan) and a flexible video naso-pharyngo-laryngoscope (VNL11-J10; PENTAX Medical). To mimic office-based transcutaneous VFI, the flexible laryngoscope was inserted via the rigid laryngoscope to visualize the vocal folds. The injector of the RLVFI device was inserted through the cervical skin (Fig. 1C and D). Hyaluronic acid (HA; Neuramis Light Lidocaine, Medytox Inc., Cheongju, Korea) was used as the filler. After the experiment, the larynx was excised from the fourth tracheal ring to the hyoid bone to examine histologic change.

\section{Histologic analysis}

The canine larynx was fixed in formaldehyde and cut axially at the level of the true vocal folds. The specimen was processed for 8 hours with a rapid hydrochloric acid decalcifier (ShandonTBD1; Thermo Fisher Scientific, Waltham, MA, USA) to remove the calcium. The specimen was embedded in paraffin blocks, sectioned, and stained (hematoxylin and eosin) for histologic analysis. Slides were scanned with Pannoramic, 250 Flash III (3DHISTECH, Budapest, Hungary). 

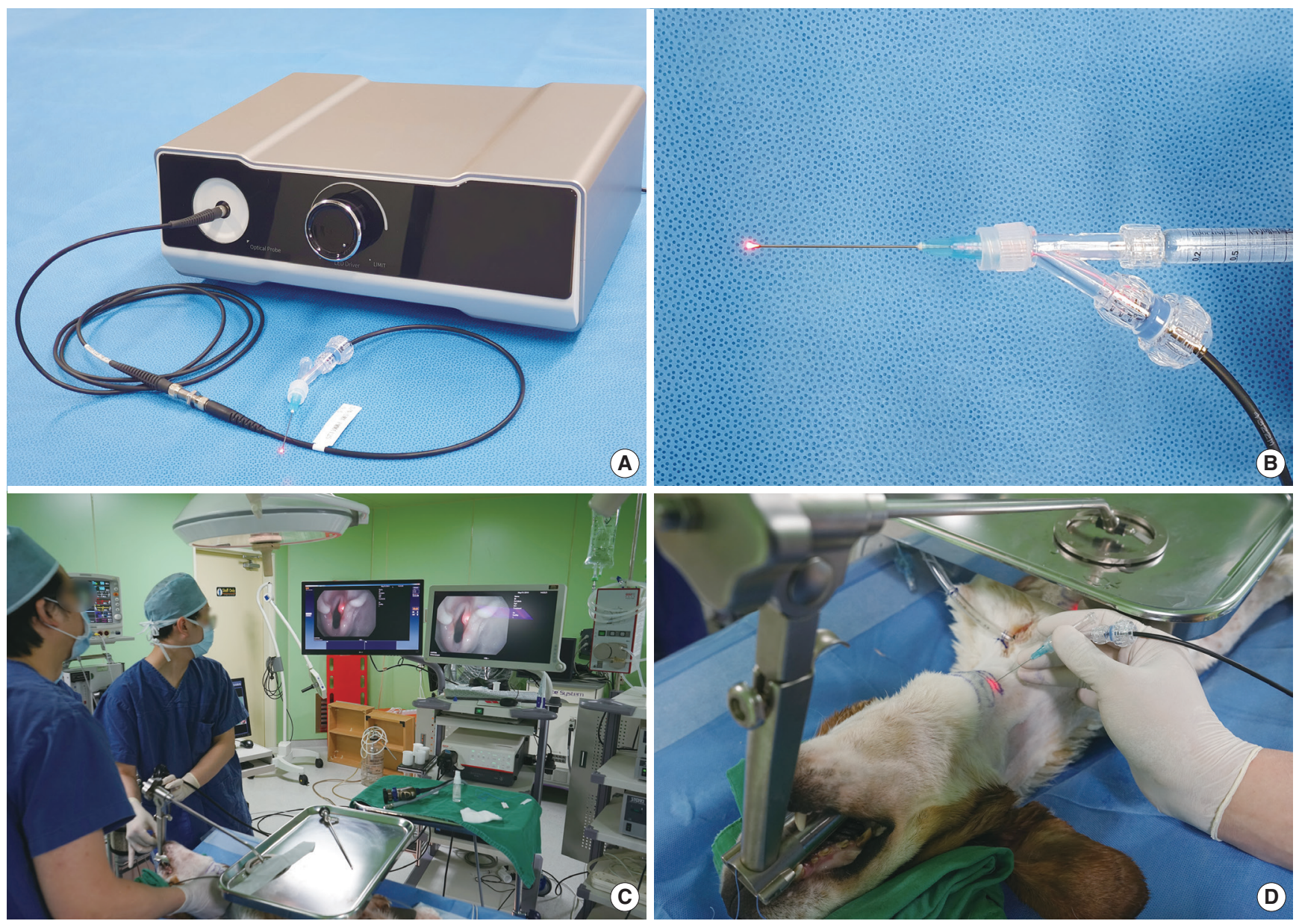

Fig. 1. (A) The device for real-time light-guided vocal fold injection (RL-VFI). (B) The injector of the RL-VFI device. (C) The experimental setting. A flexible laryngoscope was inserted via a rigid laryngoscope to visualize the vocal folds, mimicking office-based transcutaneous VFI. (D) The injector of the RL-VFI device was inserted through the cervical skin.

\section{RESULTS}

In this study, we validated various transcutaneous RL-VFI approaches in an in vivo canine model (Fig. 2). In the CT approach, the needle was inserted between the inferior border of the thyroid cartilage and the superior border of the cricoid cartilage. In the TT approach, the needle was placed above the inferior border of the thyroid cartilage and introduced perpendicular to the cartilage. In the $\mathrm{TH}$ approach, the needle was placed just above the thyrohyoid notch and inserted into the subcutaneous tissues at a downward angle.

\section{Identification of the needle tip in the CT approach}

The needle tip could be identified using the RL-VFI device in the $\mathrm{CT}$ approach, and its anatomical location in the larynx could be intuitively determined by the red light. The vocal folds were visualized using a flexible laryngoscope under a suspension rigid laryngoscope (Fig. 3A). When the injector of the RL-VFI device was gently introduced on the CT membrane, the red light was easily identified on the subglottic mucosa of the CT membrane, which helped prevent penetration into the airway (Fig. 3B). By referring to the position of the needle on the $\mathrm{CT}$ membrane, the entry point of the needle on the skin was adjusted slightly outward. As the needle was advanced gradually, the light scattered around the vocal fold, indicating that the needle had been properly introduced into the paraglottic space (Fig. 3C). To treat unilateralVFP, the material is usually injected into a point lateral to the vocal process in the thyroarytenoid muscle for deep vocal fold augmentation. Under light guidance, the needle tip could be easily positioned on the intended point lateral to the vocal process based on real-time visual feedback (Fig. 3D). The scattering and intensity of the light provided information about the depth of the needle in the vocal fold. When the needle tip approached the mucosa on the superior surface of the vocal fold, the intense light could prevent the needle from penetrating the mucosa and help to reposition it at the proper space in the deep musculature (Fig. 3E). When the needle approached the medial surface of the vocal fold, the visual information provided by the 

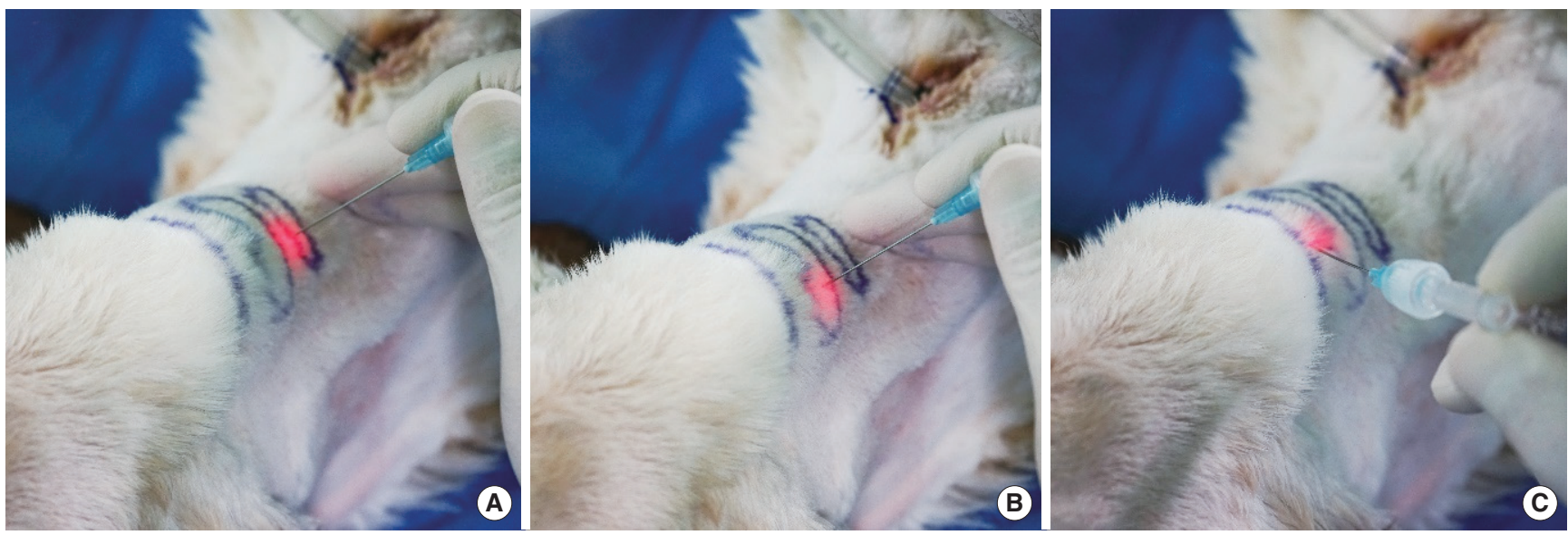

Fig. 2. Various transcutaneous approaches of the real-time light-guided vocal fold injection device in an in vivo canine model. (A) The cricothyroid approach, (B) the transthyroid approach, and (C) the transhyoid membrane approach.
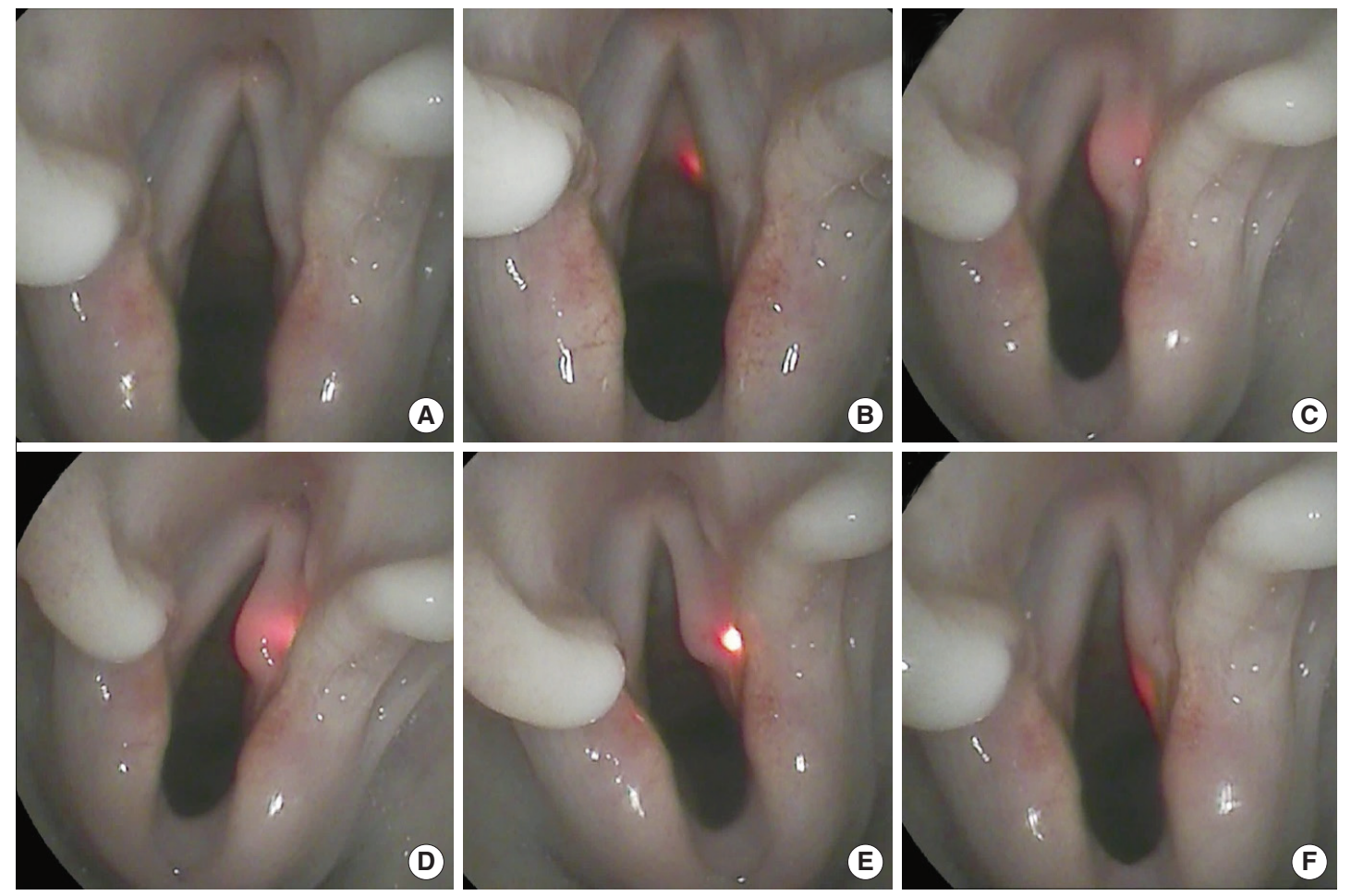

Fig. 3. Identification of the needle tip in the cricothyroid (CT) approach using the real-time light-guided vocal fold injection device. The location of the needle tip can be identified by the red light: (A) before the needle insertion, (B) on the CT membrane, (C) in the paraglottic space, (D) lateral to the vocal process, (E) on the mucosa on the superior surface of the vocal fold, and (F) on the medial surface of the vocal fold. The scattering and intensity of the light can inform the depth of the needle in the vocal fold.

light prevented inadvertent penetration into the mucosa (Fig. 3F). Depending on the degree of light scattering and transmittance, the depth of the needle was identified and controlled delicately in the vocal fold.

\section{Identification of the needle tip in the $T$ approach}

In the TT approach, the needle tip could be easily localized in the vocal fold using RL-VFI. The vocal folds were visualized using a flexible laryngoscope (Fig. 4A). The dispersed light could be identified around the vocal fold and the laryngeal ventricle after the needle was introduced through the thyroid cartilage (Fig. 4B). Under light guidance, the needle was moved into the thyroarytenoid muscle and could be located at the target point lateral to the vocal process based on visual feedback (Fig. 4C). 

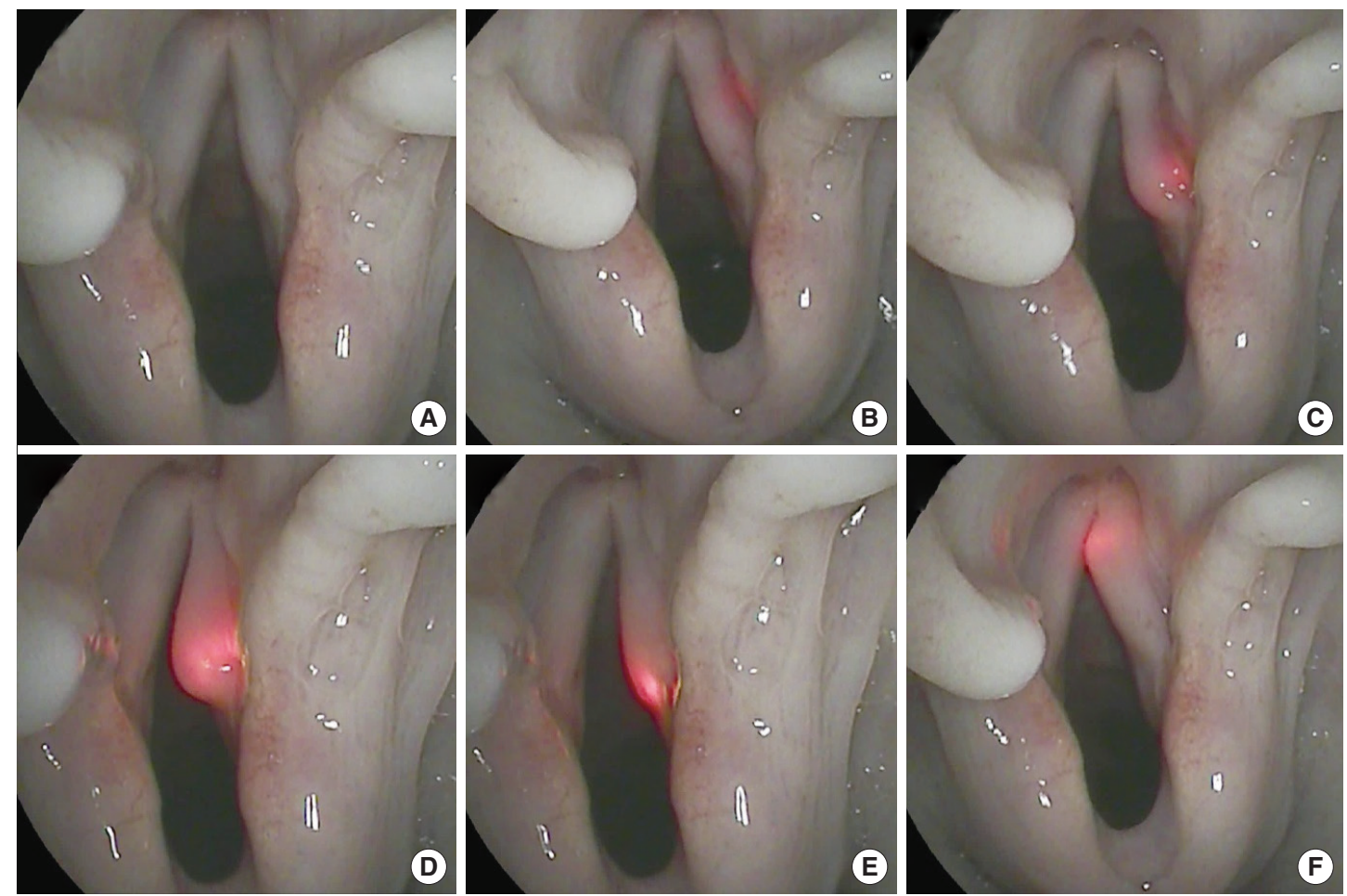

Fig. 4. Identification of the needle tip in the transthyroid approach using real-time light-guided vocal fold injection. The location of the needle tip can be identified by the red light: $(A)$ before the needle insertion, $(B)$ around the vocal fold and the laryngeal ventricle, $(C)$ lateral to the vocal process, (D) around the vocal process, (E) on the posterior mucosa, and (F) on the anterior vocal fold mucosa.

When the needle proceeded across the vocal process, the vocal process was shaded and easily identified (Fig. 4D). The visual information provided by light emission and scattering in the vocal fold demonstrated the direct orientation of the needle tip. Furthermore, the device could assist the needle tip to reach the posterior mucosa precisely, preventing unintentional penetration (Fig. 4E). The anterior vocal fold mucosa was easily accessed using the light-guided needle (Fig. 4F).

\section{Identification of the needle tip in the TH approach}

The route of the needle tip could be identified by the red light of the device during the TH approach. The vocal folds and the epiglottis were visualized using a flexible laryngoscope (Fig. 5A). The needle should be inserted to the petiole near the vocal fold through the pre-epiglottic space and the epiglottic cartilage. The precise penetration of the epiglottic mucosa is important for successfully performing VFI into the intended point. The needle was inserted through the TH membrane and was identifiable around the petiole based on the scattered light (Fig. 5B). The light was observed in a sufficiently low-lying point around the petiole, allowing the operator to insert the needle into the vocal fold at a comfortable angle (Fig. 5C). After the needle was positioned at the intended point just lateral to the petiole, it penetrated the mucosa (Fig. 5D and E). After the needle penetrated the appropriate point, it was easily inserted into the vocal fold (Fig. 5F).

\section{RL-VFI with HA via the CT approach using the device}

RL-VFI with HA was performed through the $\mathrm{CT}$ approach using the device. The illuminated needle was inserted through the CT membrane and positioned in the deep thyroarytenoid muscle of the left vocal fold (Fig. 6A and B). The injection of HA into the muscle was subsequently started under light guidance, and it medialized the left vocal fold slightly (Fig. 6C). Because HA has higher light transmittance than muscle and mucosa, the illuminated needle in the material showed stronger dispersion, which could provide information on the extent of the injectate (Fig. 6D and E). After removal of the needle, it was confirmed that the vocal folds were sufficiently medialized (Fig. 6F).

Histologic evaluation after HA injection using the RL-VFI device In the postmortem analysis, the distance from the anterior commissure (AC) to the posterior commissure was $18 \mathrm{~mm}$ and the distance from $\mathrm{AC}$ to the vocal process was $8 \mathrm{~mm}$ in the canine larynx. The diameter of the trachea was $18 \times 21 \mathrm{~mm}$ and the distance from the superior thyroid notch to the inferior thyroid notch was $17 \mathrm{~mm}$.

The hematoxylin and eosin-stained section of the extracted vocal folds showed that HA had been properly injected into the thyroarytenoid muscle of the left vocal fold (Fig. 7). There were no histological findings related to thermal damage on the tissue around the materials in the left vocal fold. 

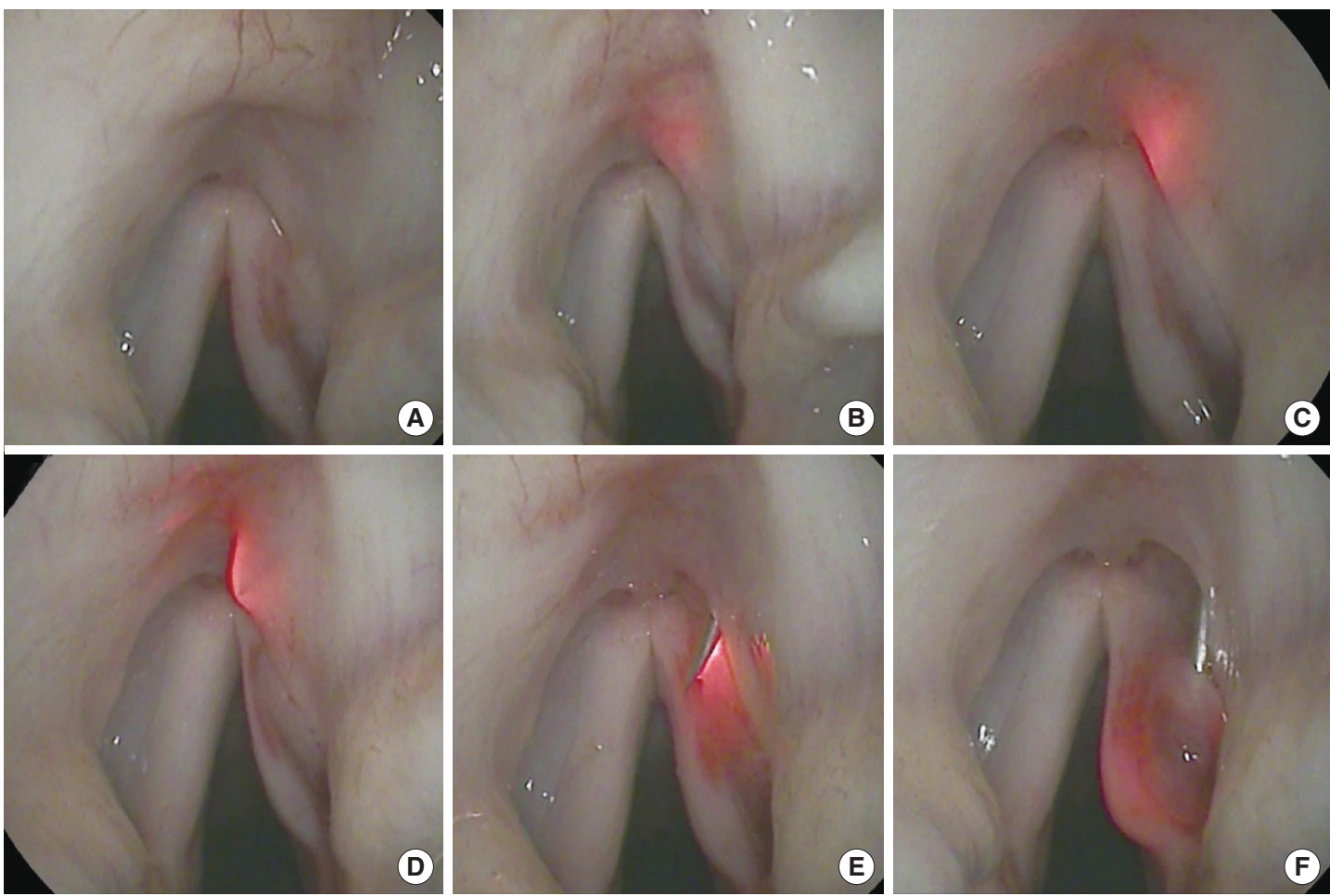

Fig. 5. Identification of the needle tip in the transhyoid approach using real-time light-guided vocal fold injection. The location and the route of the needle tip can be identified by the red light: $(A)$ before the needle insertion, $(B)$ in the pre-epiglottic space, $(C, D)$ on the mucosa of the petiole, (E) during the penetration of the mucosa, and (F) during the insertion of the needle into the vocal fold.
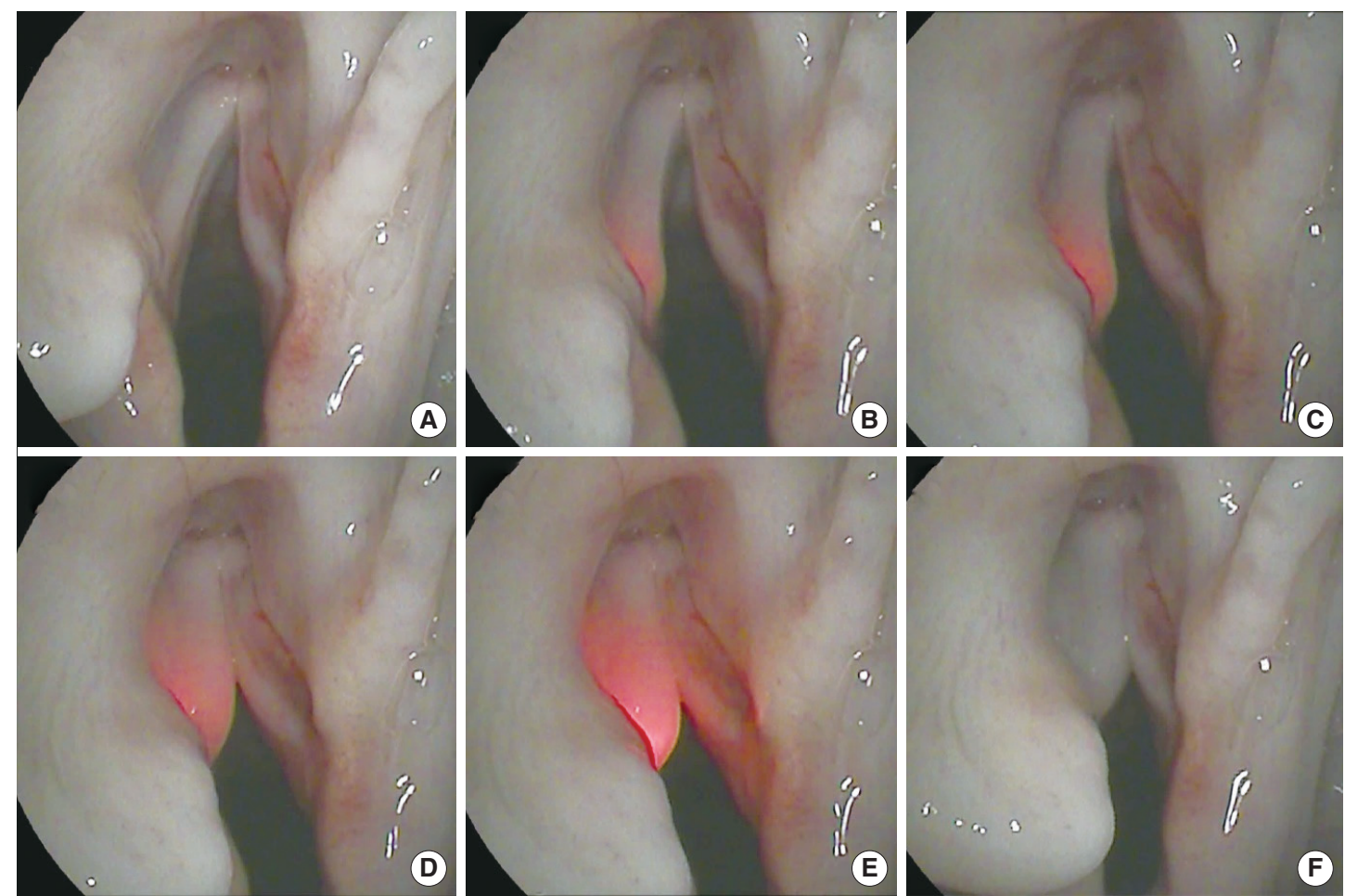

Fig. 6. Real-time light-guided vocal fold injection with hyaluronic acid $(H A)$ in the cricothyroid approach: $(A)$ before the needle insertion, $(B)$ placement of the illuminated needle in the deep thyroarytenoid muscle, and (C-E) during injection of HA into the muscle. HA has a higher light transmittance than muscle and mucosa; therefore, the illuminated needle in the material shows stronger dispersion, which can provide information on the extent of the injectate. (F) After removal of the needle, it could be confirmed that the vocal folds was sufficiently medialized. 


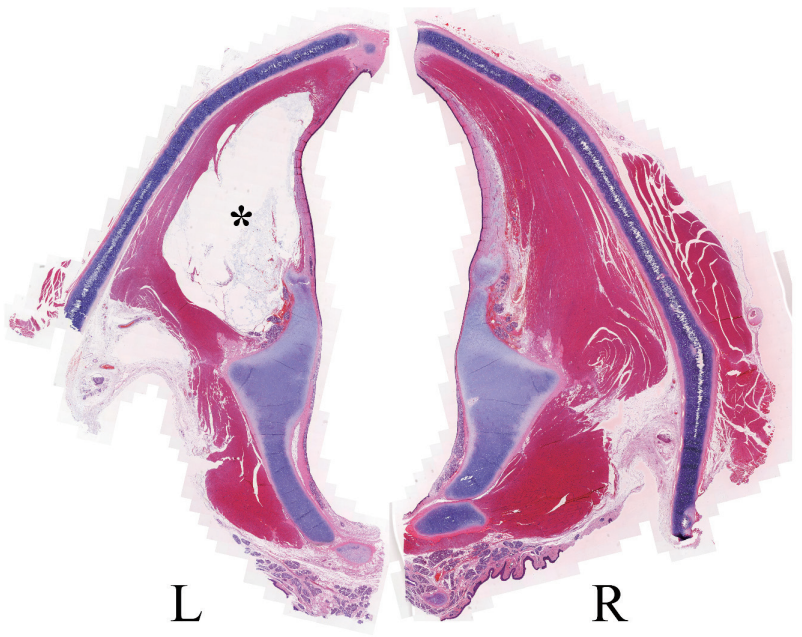

Fig. 7. H\&E-stained section of the extracted vocal folds showed that hyaluronic acid (asterisk) had been properly injected into the thyroarytenoid muscle of the left vocal fold. There were no histological findings related to thermal damage on the tissue around the materials in the left vocal fold. L, left; R, right.

\section{DISCUSSION}

The CT approach has the advantages of a low complication rate, suitability as an office-based procedure, and good patient compliance compared to other mucosa-penetrating procedures such as the transoral or TH approaches [2,6,7]. During the CT approach, inadvertent penetration of the mucosa can lead to coughing, bleeding, failure, cessation or delay in the procedure, and low patient satisfaction $[2,4,5,12,13]$. In our study, the RLVFI device provided the precise location of the needle by light guidance and could intuitively prevent inadvertent penetration in the CT approach. Usually, the position of the needle can be indirectly identified by conventional maneuvers, such as palpation of the CT membrane and alteration of the vocal fold configuration. However, alteration of the vocal fold configuration can only be noticed when the needle enters into the thyroarytenoid muscle $[2,7,12]$. Without an anatomical or spatial orientation correlating external landmarks with internal laryngeal structures, it can be difficult to insert the needle into the muscle in a few attempts within a short time $[4,5,12]$. The RL-VFI device can be useful to identify the needle during the blind pathway from the skin to the thyroarytenoid muscle $[4,5]$. The injector of the device could radiate the scattered light and provide visual information about the direction and depth when it enters into the deep paraglottic space before reaching the thyroarytenoid muscle. The location and depth of the injection point can considerably affect the final configuration of the vocal fold after VFI $[4,5,14]$. In the CT approach, precise injection is challenging due to the invisibility of the needle tip, as is widely known, and no existing techniques provide information on the depth orientation of the needle. RL-VFI could provide information about the anterior-to-posterior or medial-to-lateral position, as well as the depth orientation, by alterations of the intensity and scattering of the light $[4,5]$.

Although the CT approach has advantages and is preferred in South Korea, a previous history of surgery or obesity can make it very difficult to perform $[1,6]$. The TT or TH approaches are useful techniques that can serve as alternative options when the $\mathrm{CT}$ approach is not possible $[1,6,8]$. For instance, the TT approach, in which the needle is inserted perpendicular to the thyroid cartilage, is very useful in small larynges with little ossification $[2,6,11]$. However, the ideal direction of the needle varies according to the insertion point. The possibility of penetration is higher than in the CT approach because the needle route is more perpendicular to the vocal fold [6-8,12]. In the in vivo canine model used in this study, the illuminated needle was very helpful for preventing the penetration of the vocal fold mucosa. The RL-VFI device could lead the needle to the intended point with spatial orientation by real-time visual feedback.

The disadvantages of the TH approach include the inability to access the anterior vocal fold in small larynges, the inability to access the posterior vocal fold in large larynges, and the difficulty in passing the needle through a calcified TH membrane $[1,2,7]$. These disadvantages result from the long pathway of this approach, which limits the range of movement of the needle. There is a potential need to pass the needle into the larynx multiple times, which can cause bleeding, gagging, or the cough reflex $[2,4,8-10]$. The optimal penetration point can vary depending on the target location of the vocal fold (anterior or posterior). To overcome these current limitations, it is important to set an optimal point before penetrating the mucosa of the epiglottis $[4,9,13]$. In this study, the illuminated needle of the device provided visual information on the position of the needle before penetrating the mucosa. This information may be helpful for determining the optimal penetration point and reducing the number of trial insertions in the TH approach.

Since Seifert and Hirst introduced the CT approach a century ago, advances in endoscopic technology and the development of biocompatible materials have accelerated the refinement of this technique $[1,2,4,7]$. However, there have been few signs of progress in the technical aspect, and most laryngologists still depend on Hirano's classical maneuvers to identify the needle position: palpation of the $\mathrm{CT}$ membrane and the examination of distortion and subtle movement of the vocal folds during needle insertion $[4,14]$. These maneuvers have been the keystone of successful VFI through the CT approach over the past decades. Nonetheless, these maneuvers fundamentally require extensive clinical experience and therefore have a steep learning curve $[4,12,14]$. Several attempts have been made to improve the CT approach technically. Jin et al. [16] suggested that anatomical references regarding the $\mathrm{CT}$ approach may provide guidelines and facilitate a deeper understanding of the procedure, eventually leading to precise access to the vocal fold. However, it is dif- 
ficult to solve the current problems facing this technique using only the image-guided approach because of the inter-individual variation in laryngeal anatomy [4,5]. Hoffman et al. [14] suggested a breakthrough technique for transillumination of the vocal folds, which utilized visual feedback during the procedure. However, the fiber-optic cable must be removed before injection and this removal-and-reinsertion technique is difficult to apply in real office-based practice [4,5].

RL-VFI was conceptualized as a technique that allows simultaneous injection under precise needle localization by light guidance [4].The RL-VFI device was developed and its technical feasibility was validated in an ex vivo canine model simulating the setting of office-based VFI [5]. However, in actual patients, the needle passes through more complex anatomical layers of the skin, the subcutaneous fat, the strap muscles, the CT membrane, the paraglottic space, and the thyroarytenoid muscle $[4,5]$. It is more challenging to determine the optimal entry point because the external anatomical landmarks might be obscure $[5,13,14]$. In this study, RL-VFI was feasible in an in vivo canine model with all anatomical structures present, using the $\mathrm{CT}$ approach in addition to the TT and the TH approaches. VFI of HA could be simultaneously performed with no additional manipulation using the RL-VFI device. The dispersed light provided information about the extent of injected materials due to its transparency and was even helpful for reinjections.

The canine model used in this study weighed $13 \mathrm{~kg}$, and it had a relatively small larynx with an 8-mm-long vocal fold, considering that the average length of the vocal fold is $13.5 \mathrm{~mm}$ and $15.3 \mathrm{~mm}$ in adult men and women, respectively [17]. However, the success of injections into this small larynx indicates that delicate and precise manipulation is possible with the aid of the device.

In modern laryngology, VFI has developed mainly into an awake in-office procedure $[1,2,12]$. In our study, the animal was inevitably sedated under general anesthesia, so the procedure could not completely simulate awake in-office practice. Therefore, a clinical trial is mandatory to establish the clinical safety and effectiveness of the RL-VFI device. We plan to carry out a pilot clinical study to apply the device for office-basedVFI in the future.

After the introduction of RL-VFI in the clinical field, we anticipate that various aspects of VFI will be improved. RL-VFI will improve the precision and safety of VFI for the treatment of unilateral VFP. The RL-VFI device will facilitate customized VFI according to the shape of the paralyzed vocal fold, as the clinician controls the dose and location of injection. It will also help prevent inadvertent penetration and mis-injection into the superficial layer, which can lead to disastrous problems for the voice [18]. From an educational perspective, RL-VFI will help trainees to understand the route of the needle during transcutaneous VFI and learn it quickly. A training program using RL-VFI would be helpful for trainees to practice transcutaneous VFI before at- tempting it on actual patients [19]. When counseling patients, it can be difficult to explain the concept of transcutaneousVFI due to the invisibility of the needle. RL-VFI will help patients to understand the procedure intuitively and be reassured.

RL-VFI was found to be feasible and safe in an in vivo canine model, providing precise localization and visual-motor feedback. This study demonstrated the feasibility of RL-VFI in an in vivo canine model, establishing the rationale for an upcoming clinical trial. The clinical application of RL-VFI is expected to improve the safety and precision of VFI.

\section{CONFLICT OF INTEREST}

The corresponding author (WC) is the inventor of the device. Pusan National University and Pusan National University Hospital hold the patents related to the device, granted by the Korean Intellectual Property Office (KR101699229B1), the Patent Cooperation Treaty (WO2017039193A1), and United States Patent and Trademark Office (US15/536,419). The authors have no other funding or financial relationship to disclose.

\section{ACKNOWLEDGMENTS}

This study was supported by grant no. 02-2020-009 from the SNUBH Research Fund.

\section{ORCID}

Hee Young Son https://orcid.org/0000-0002-2845-1423

Sanghoon Kim https://orcid.org/0000-0001-6186-0790

RamlaTalib Mohammad https://orcid.org/0000-0003-3257-4458

Gene Huh

https://orcid.org/0000-0003-4487-1361

Hyojin Kim https://orcid.org/0000-0001-9201-8328

Woo-Jin Jeong https://orcid.org/0000-0001-8577-6964

Wonjae Cha https://orcid.org/0000-0001-7292-9474

\section{AUTHOR CONTRIBUTIONS}

Conceptualization: WC. Data curation: SK, RTM, GH. Formal analysis: RTM, GH. Methodology: HK, WC. Project administration:WC. Visualization \& Writing-original draft: HYS, WC. Writing-review \& editing: HYS, WJJ,WC.

\section{REFERENCES}

1. Rosen CA, Amin MR, Sulica L, Simpson CB, Merati AL, Courey MS, et al.Advances in office-based diagnosis and treatment in laryngolo- 
gy. Laryngoscope. 2009 Nov;119 Suppl 2:S185-212.

2. Mallur PS, Rosen CA.Vocal fold injection: review of indications, techniques, and materials for augmentation. Clin Exp Otorhinolaryngol. 2010 Dec;3(4):177-82.

3. Kwon TK, Buckmire R. Injection laryngoplasty for management of unilateral vocal fold paralysis. Curr Opin Otolaryngol Head Neck Surg. 2004 Dec;12(6):538-42.

4. Cha W, Ro JH, Wang SG, Jang JY, Cho JK, Kim GH, et al. Development of a device for real-time light-guided vocal fold injection: a preliminary report. Laryngoscope. 2016 Apr;126(4):936-40.

5. Cha W, Ro JH, Yang SC, Choi CJ, Yang I, Kang H, et al. Real-time light-guided vocal fold injection: ex vivo feasibility study in a canine model. Laryngoscope. 2019 Apr;129(4):935-42.

6. Lee SW, Kim JW, Koh YW, Shim SS, Son YI. Comparative analysis of efficiency of injection laryngoplasty technique for with or without neck treatment patients: a transcartilaginous approach versus the cricothyroid approach. Clin Exp Otorhinolaryngol. 2010 Mar;3(1): 37-41.

7. Chhetri DK, Jamal N. Percutaneous injection laryngoplasty. Laryngoscope. 2014 Mar;124(3):742-5.

8. Achkar J, Song P, Andrus J, Franco R Jr. Double-bend needle modification for transthyrohyoid vocal fold injection. Laryngoscope. 2012 Apr;122(4):865-7.

9. Amin MR. Thyrohyoid approach for vocal fold augmentation. Ann Otol Rhinol Laryngol. 2006 Sep;115(9):699-702.

10. Zeitler DM,Amin MR. The thyrohyoid approach to in-office injection augmentation of the vocal fold. Curr Opin Otolaryngol Head Neck Surg. 2007 Dec;15(6):412-6.

11. Rees CJ, Mouadeb DA, Belafsky PC. Thyrohyoid vocal fold augmentation with calcium hydroxyapatite. Otolaryngol Head Neck Surg.
2008 Jun;138(6):743-6

12. Pearson W, Hutchinson CT, Noordzij JP.Accessing the vocal folds by transcutaneous injection. Clin Anat. 2010 Apr;23(3):270-6.

13. Clary MS, Milam BM, Courey MS. Office-based vocal fold injection with the laryngeal introducer technique. Laryngoscope. 2014 Sep; 124(9):2114-7.

14. Hoffman HT, Dailey SH, Bock JM, Thibeault SL, McCulloch TM. Transillumination for needle localization in the larynx. Laryngoscope. 2015 Oct;125(10):2341-8.

15. Statistics Korea, National Health Insurance Corporation Healthcare Bigdata Hub, Health Insurance Review \& Assessment Service. Sta tistics of vocal fold injection, thyroplasty and arytenoid adduction using operation code by O1264, O1265 and O1266 [Internet].Wonju: Health Insurance Review \& Assessment Service; 2017 [cited 2020 Oct 6]. Available from: http://opendata.hira.or.kr/op/opc/olapDiagBhvInfo.do.

16. Jin SM, Park CY, Lee JK, Ban JH, Lee SH, Lee KC. Transcutaneous injection laryngoplasty through the cricothyroid space in the sitting position: anatomical information and technique. Eur Arch Otorhinolaryngol. 2008 Mar;265(3):313-9.

17. Su MC, YehTH,Tan CT, Lin CD, Linne OC, Lee SY. Measurement of adult vocal fold length. J Laryngol Otol. 2002 Jun;116(6):447-9.

18. Chheda NN, Rosen CA, Belafsky PC, Simpson CB, Postma GN. Revision laryngeal surgery for the suboptimal injection of calcium hydroxylapatite. Laryngoscope. 2008 Dec;118(12):2260-3.

19. Kim J, Seo HS, Na HS, Son HY, Lee DK, Jeong WJ, et al. Real-time light-guided vocal fold injection as a simulation-based training tool. Auris Nasus Larynx. 2020 Aug 12 [Epub]. https://doi.org/10.1016/ j.anl.2020.07.011. 